\title{
Retrospective Studies of Gastric Cancer with Hepatic Metastases
}

\author{
JINRYO TAKEDA, KENJI YASUMOTO, SHOJIRO YANO, TAKAHO TANAKA, \\ KIKUO KOUFUJI, KEN HASHIMOTO AND TERUO KAKEGAWA
}

Department of Surgery, Kurume University School of Medicine, Kurume, 830 Japan

Received for publication September 5, 1990

\begin{abstract}
Summary: From 1979 to 1988, a total of 1265 cases of gastric cancer were admitted to our Institute. Of these, 80 cases $(6.3 \%)$ involved hepatic metastasis. Thirty-eight cases (47.3\%) underwent gastrectomy (24 distal, 2 proximal, 11 total and one partial). Of these 38 cases, 7 underwent a combined resection of a simultaneous metastatic hepatic tumor. A total of $19(67.9 \%)$ of the 28 cases in $\mathrm{H}_{1}, 6(37.5 \%)$ of the 16 cases in $\mathrm{H}_{2}$, and $13(36.1 \%)$ of the 36 cases in $\mathrm{H}_{3}$ underwent gastrectomy. Hepatic metastasis occurred most frequently (18.2\%) in Borrmann type $1,5 \%$ in type $2,14.9 \%$ in type 3 and $8.7 \%$ in type 4. Of all 593 resected advanced cases, there were 283 differentiated type, with a hepatic metastasis rate of $14.5 \%$, and 310 undifferentiated type with $7.1 \%$ rate of hepatic metastasis. In $\mathrm{H}_{1}$ and $\mathrm{H}_{2}$, the prognosis after primary gastric cancer resection was better than for unresected cases. Moreover, there have been reports recently of long-term survival using continuous hepatic arterial infusion of anti-cancer durgs, and of combined resection of the metastatic hepatic tumor after curative gastrectomy. Therefore we should not give up these $\mathrm{H}_{1}$ and $\mathrm{H}_{2}$ patients.
\end{abstract}

Key words: gastric cancer - hepatic metastasis - gastrectomy - combined resection - mean survival time

\section{Introduction}

The prognosis of gastric cancer has improved year by year due to the increased early detection of gastric cancer and to recent advancement in various techniques for treatment. However, gastric cancer still remains the leading cause of cancer death in Japan. Here we report a retrospective study of 80 gastric cancer patients with hepatic metastasis reviewed with special reference to the factors contributing to the poor prognosis for such patients.

\section{Patients and Methods}

From 1979 to 1988 , a total of 1265 cases with gastric cancer were admitted to our Department of Surgery. Of these, 80 cases (6.3\%) involved hepatic metastasis, with an age range of 32-84 years (mean 65.1 years) and a male to female ratio of $4.3: 1$. The 80 cases were subdivided according to the resectability of the primary gastric cancer into a resectable group and a non-resectable group.

The 38 cases $(47.5 \%)$ that underwent resection involved 24 distal, 2 proximal, 11 total and one partial gastrectomy. Of these, 7 underwent a combined resection of a simultaneous metastatic hepatic tumor. Of the 42 cases $(52.5 \%)$ in the nonresectable group, 11 underwent exploratory laparotomy, 9 underwent a bypass operation, while the other 22 included 16 non-operation, 3 feeding gastrostomy, 
and 4 Celestin prosthetic tube for cardia stenosis.

According to the criteria set by the Japanese Research Society for Gastric Cancer (1981), the cases of hepatic metastasis can be subdivided into the four groups, $\mathrm{H}_{0}, \mathrm{H}_{1}, \mathrm{H}_{2}$ and $\mathrm{H}_{3} . \mathrm{H}_{0}$ : No liver metastasis. $\mathrm{H}_{1}$ : Metastasis limited to one lobe. $\mathrm{H}_{2}$ : A few scattered metastatic sites in both lobes. $\mathrm{H}_{3}$ : Numerous scattered metastatic sites in both lobes. Of the 28 cases in $\mathrm{H}_{1}, 19$ (67.9\%) were resected primary gastric cancers, involving $7 \mathrm{com}$ bined resections of the metastatic liver tumors and 12 gastrectomy only. The other 9 cases $(32.1 \%)$ in $\mathrm{H}_{1}$ underwent no resection of the gastric cancer and no resection of the metastatic tumor.

Of the 16 cases in $\mathrm{H}_{2}, 6$ (37.5\%) underwent gastrectomy only, and of the 36 cases in $\mathrm{H}_{3}, 13$ (36.1\%) underwent gastrectomy only (Table 1 ).

TABLE 1

Primary Gastric Cancer Resection and $H$-Degree

\begin{tabular}{c|rc|c}
\hline & Resected & Unresected & Total \\
\hline $\mathrm{H}_{1}$ & $19(67.9)$ & $9(32.1)$ & $28(35.0)$ \\
$\mathrm{H}_{2}$ & $6(37.5)$ & $10(62.5)$ & $16(20.0)$ \\
$\mathrm{H}_{3}$ & $13(36.1)$ & $23(63.9)$ & $36(45.0)$ \\
\hline Total & \multicolumn{1}{|c}{38} & 42 & $80(100)$ \\
\hline \multicolumn{2}{l}{}
\end{tabular}

The gastric cancer location, the degree of hepatic metastasis, and the total number and rate of gastrectomy with hepatic metastasis are summarized in Table 2. Hepatic metastasis was most frequently observed in cases with $\mathrm{C}$ region (upper third of the stomach) primary gastric cancer. The locations of the gastric cancer of cases in the $\mathrm{H}_{1}$ group are summarized in Fig. 1. The relation between macroscopic classification by Borrmann and hepatic metastasis rates is summarized in Fig. 2. Hepatic metastasis occurred most frequently (18.2\%) in Borrmann type $1,5 \%$ in type $2,14.9 \%$ in type 3 and $8.7 \%$ in Borrmann type 4 . The relation between histological classification and hepatic metastasis for resected advanced cases is summarized in Fig. 3. Of all 593 resected advanced cases, there were 283 of the differentiated type, with a hepatic metastasis rate of $14.5 \%$, and 310 of the undifferentated type with a rate of $7.1 \%$.

Frozen specimens of gastric adenocarcinoma $(n=80)$ and hepatic metastasis $(\mathrm{n}=2)$ were investigated immunohistochemically to examine for the existence of laminin (LM) in the tissue. LM was $40 \%$ positively stained in the differentiated type (Table 3 ).

Prognosis in $\mathrm{H}(+)$ cases and cancer stage including disseminating metastasis to the peritoneum $(\mathrm{P})$, serosal invasion $(\mathrm{S})$ and lymphnode metastasis (N) are sum-

TABLE 2

Gastric Cancer Location and $H$-Degree

\begin{tabular}{|c|c|c|c|c|c|}
\hline $\begin{array}{l}\text { H-Degree } \\
\text { Location } \\
\end{array}$ & $\mathrm{H}_{1}$ & $\mathrm{H}_{2}$ & $\mathrm{H}_{3}$ & $\begin{array}{c}\text { Hepatic } \\
\text { Metastasis }\end{array}$ & $\begin{array}{c}\text { Hepatic } \\
\text { Metastasis } / \text { Resected } \\
\text { advanced No. }\end{array}$ \\
\hline $\mathrm{C}$ & 11 & 6 & 17 & $34 / 293(11.6)$ & $34 / 194(17.5)$ \\
\hline M & 4 & 4 & 11 & $19 / 488(3.9)$ & $19 / 225(8.4)$ \\
\hline $\mathrm{A}$ & 13 & 6 & 8 & $27 / 394(6.9)$ & $27 / 213(12.7)$ \\
\hline Total & 28 & 16 & 36 & $80 / 1175(6.8)$ & $80 / 632(12.7)$ \\
\hline
\end{tabular}




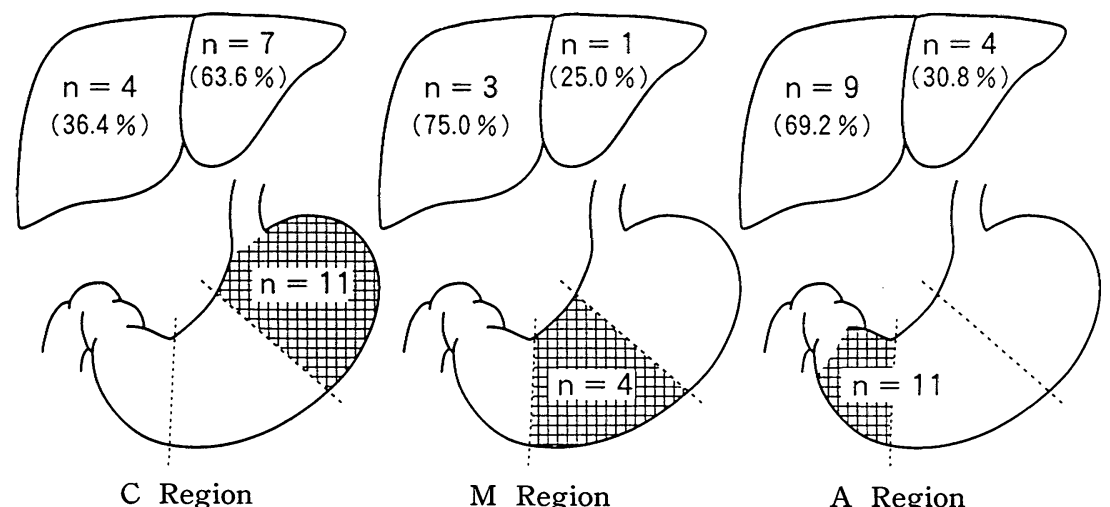

Fig. 1 Gastric cancer location and hepatic metastases in $\mathrm{H}_{1}$

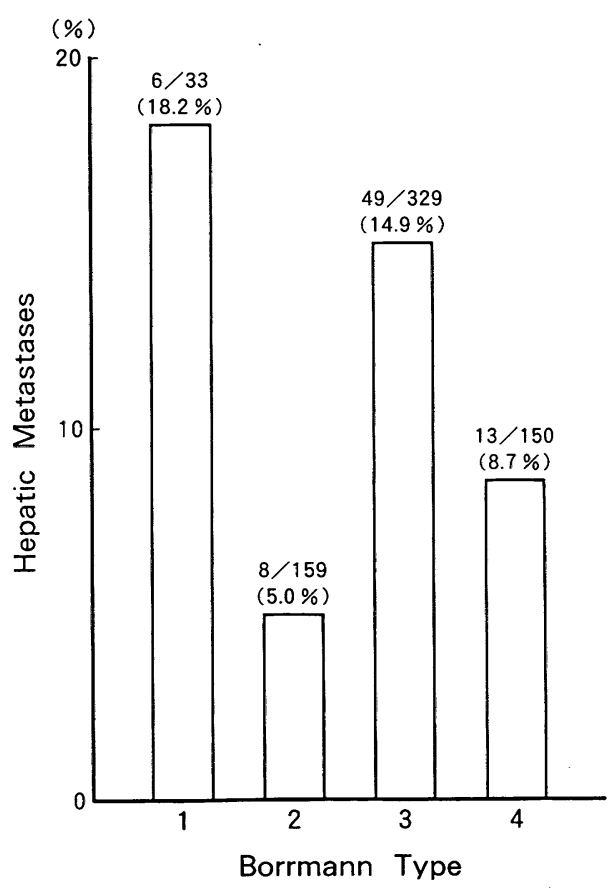

Fig. 2 Macroscopic classification (Borrmann type) and hepatic metastases

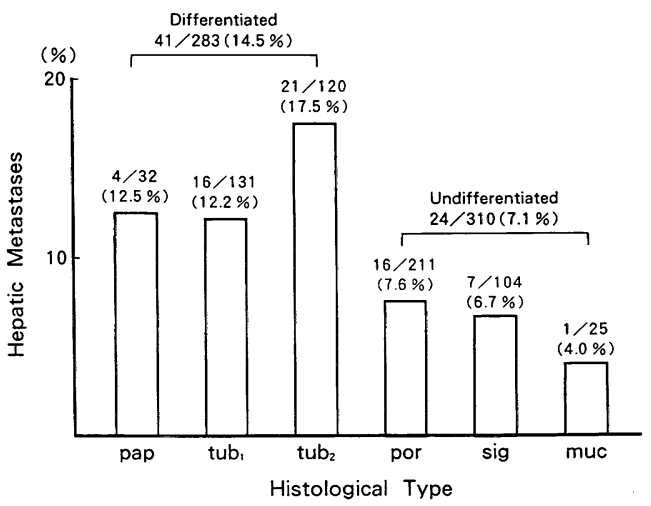

Fig. 3 Hepatic metastases by histological classification (No. of hepatic metastases/ No. of resected advanced cancer)

TABLE 3

Rate of positive laminin staining by histological type

\begin{tabular}{|c|c|c|c|}
\hline tub$_{1}$ & $18 / 36$ & $(50.0)-$ & \\
\hline $\begin{array}{l}* \text { Resected Hepatic } \\
\text { Metastases }\end{array}$ & $2 / 2$ & & $20 / 50(40.0)$ \\
\hline tub2 $_{2}$ & $2 / 14$ & (14.3) - & \\
\hline por & $0 / 19$ & (0) - & \\
\hline sig & $0 / 9$ & (0) & $0 / 30$ \\
\hline muc & $0 / 2$ & $(0) \perp$ & \\
\hline
\end{tabular}




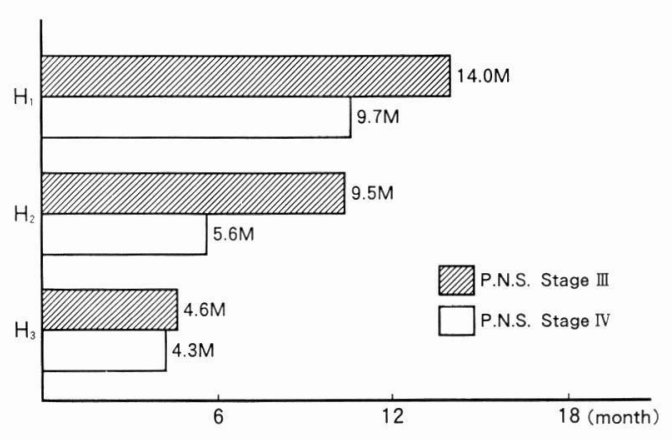

Fig. 4 Survival time by P.N.S. stage and by $\mathrm{H}$-Factor

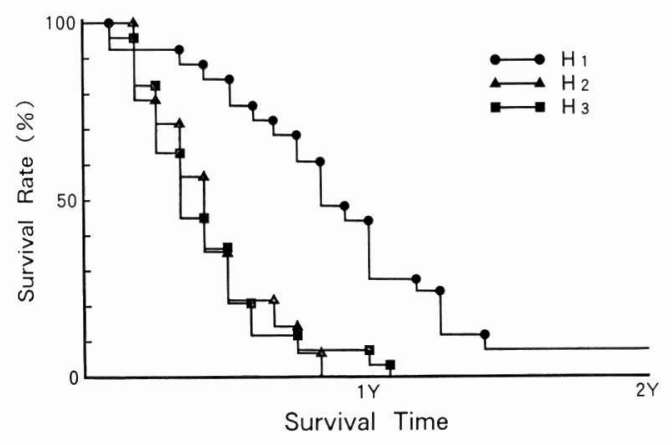

Fig. 5 Survival time according to $\mathrm{H}$ Degree

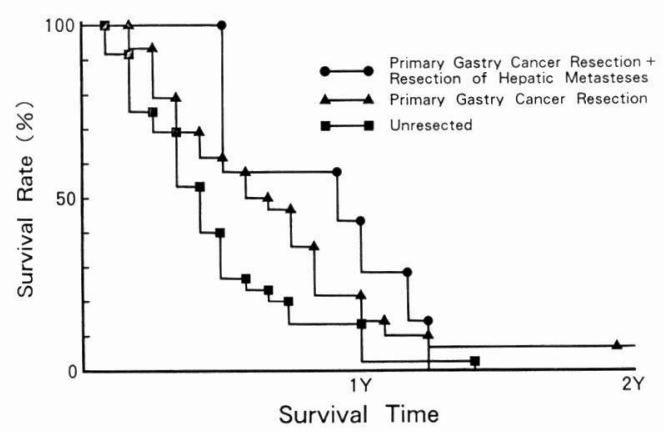

Fig. 6 Survival time according to operation marized in Fig. 4. In $\mathrm{H}_{1}$, the mean survival time was 14 months in stage III $\left(\mathrm{P}_{0}\right.$, $\left.\mathrm{N}_{2}, \mathrm{~S}_{2}\right)$ and 9.7 months in stage IV $\left(\mathrm{P}_{1^{-}}\right.$, $\mathrm{N}_{3}, 4, \mathrm{~S}_{3}$ ).

While in $\mathrm{H}_{3}$, the mean survival time was 4.6 months in stage III and 4.3 months in stage $\mathrm{IV}$.

Cancer prognosis and the degree of liver metastasis are summarized in Fig. 5, showing enhanced survival for those in $\mathrm{H}_{1}$.

The short-term prognosis and survival rates for simultaneous combined resection, gastric only resection, and non-resection, are summarized in Fig. 6.

\section{Discussion}

The liver, by virtue of its location, blood supply, anatomy and other unknown factors, provides a major secondary location for any malignant tumor of the stomach. From a retrospective study of 80 cases of gastric cancer with hepatic metastasis, the incidence of differentiated type $(14.5 \%)$ was higher than of undifferentiated types $(7.1 \%)$ histologically.

On macroscopic classification, Borrmann type 1 was most frequently observed (18.2\%). Koga et al. (1987) have reported that recurrence in the liver develops more frequently in cases of primary gastric cancer of Borrmann type 2 .

The incidence of hepatic matastasis was higher in patients with subserosal venous invasion than in those with only submucosal venous invasion. The prognosis for gastric cancer with hepatic metastasis is quite poor - nearly all are dead within 2 years of diagnosis. Gastric cancer patients with a rapidly growing tumor and extensive hepatic metastases generally die within a few months. Gastric cancer patients with hepatic metastasis have been thought of as inoperable and the treatment for most of them has often been laparotomy alone. However, the re- 
cent rapid progress in surgical nutrition, preoperative diagnosis for the extent of hepatic metastasis using US, CT, and MRI, and postoperative care have enabled the non-combined curative resectiom of the primary gastric cancer safely, which suggests improved chances for survival after a combined resection. There have been some studies on the prognostic significance of primary tumor resection for gastric cancer patients with hepatic metastasis. However, hepatic metastasis in cancer patients frequently occur together with peritoneal dissemination $(\mathrm{P})$, juxta lymphnode metastasis $\left(\mathrm{N}_{3} \mathrm{~N}_{4}\right)$, and cancer invasion to the adjacent organs $\left(\mathrm{S}_{3}\right)$. Okuyama et al. (1988) have reported that among 293 gastric cancer patients with hepatic metastasis, $118(40.3 \%)$ had peritoneal dissemination $\left(\mathrm{P}_{(+)}\right)$, showing a $\mathrm{P}_{(+)}$factor was common in those patients.

The prognostic significance of resection of the primary gastric tumor in patients with hepatic metastasis was observed in $\mathrm{H}_{1}$ plus $\mathrm{P}_{0} \mathrm{~N}_{2} \mathrm{~S}_{2}$ (stage III) with mean survival time of 14 months. However, in $\mathrm{H}_{3}$, when $\mathrm{P}, \mathrm{N}$, and $\mathrm{S}$ factors were at stage III, then the mean survival time was only 4.6 months, and at stage $\mathrm{IV}$, it was 4.3 months. In $\mathrm{H}_{2}$, the mean survival time was 9.5 months in P.N.S. stage III, and 5.6 months in P.N.S. stage IV, in our present study. Okuyama et al. (1988) have reported in the $\mathrm{P}_{0} \mathrm{H}_{+}$ gastric cancer patients, that primary tumor resection plus postoperative chemotherapy was significantly better for prognosis than resection alone or no resection. However, there was no significant difference in prognosis between the group with resection plus postoperative chemotherapy and the no-resection group in $\mathrm{P}_{(+)} \mathrm{H}_{(+)}$gastric cancer patients.

Metastasis in the liver are only rarely solitary. Typically there are multiple in dependent irregular nodules of varying sizes scattered throughout both lobes. However, some recent reports of pro- longed survival can be found in the literature of primary gastric cancer resection with combined continuous intra-arterial infusion of anti-tumor drugs (Fujimoto et al. 1987) and combined partial resection of the metastatic tumor, (Tanemura et al. 1990). In our 7 cases of combined resection of the hepatic metastasis, we could not obtain such a long survival time. However, one gastric cancer patient with solid hepatic metastasis, who underwent total gastrectomy for Borrmann type 2, and 13 months later underwent partial resection of the metastatic liver tumor, is now disease-free 2 years and 5 months after the hepatectomy. The indication for the resection of the primary gastric tumor only in cases with hepatic metastasis has been thought to be only for the control of gastric bleeding or gastric stenosis. However, in $\mathrm{H}_{1}$ and $\mathrm{H}_{2}$, the prognosis for primary gastric cancer resection is generally better than for unresected cases, and there are some recent reports of long term survival using continuous hepatic arterial infusion of anti-cancer drugs, or of combined resection of the metastatic liver tumor after curative gastrectomy. Therefore we should not give up these unfortunate patients.

\section{References}

Fujimoto, S., Serestha, R. D., Kasanuki, J., Kokubun, M., Ichiki, N., Miyazaki, A., Ohta, M. and OKuі, K. (1987). Gastric cancer with synchronous unresectable hepatic metastasis and a positive response to chemotherapy. Jpn. J. Surg. 17, 307-311.

Japanese Research Society for Gastric Cancer. The general rules for the gastric cancer study in surgery and pathology. Jpn. J. Surg. (1981). 11, 127-139.

Koga, S., Takebayashi, M., Kaibara, N., Nishidoi, H., Kimura, O., Kawasumi, H. and Makino, M. (1987). Pathological characteristics of gastric cancer that develop hematogenous recurrence, with special reference to the site of recur- 
rence. J. Surg. Oncol. 36, 239-242.

Okuyama, K., Onoda, S., Tohnosu, N., Yаmamoto, Y., Hanaoka, A., Koide, Y., Seki, S., Hara, T. and Isono, K. (1988). The prognostic significance of resection of primary tumor in gastric and colorectal cancer patients with synchronous liver metastasis. Jpn. J. Surg. 18, 7-17.

Okuyama, K., Isono, K., Juan, I., Onoda, S., OchiaI, T., Yамамото, Y., KoIde, Y. and Sатон, $\mathrm{H}$.
(1985). Evaluation of treatment for gastric cancer with liver metastasis. Cancer 55, 2498-2505.

Tanemura, H., Saji, S., Tanaka, N., Ito, T., Oshita, H., Fukata, D., Furuta, T., Azuma, S., Miya, K., Kunieda, K., Takao, F., Sugiyama, Y., Yoshida, A. and Shimokawa, K. (1990). Evaluation of surgical treatment for gastric cancer with liver metastasis. Jpn. J. Gastroenterol. Surg. 23, 1036-1463. 